\title{
Weakly nonlinear quantum transport: An exactly solvable model
}

\author{
Jian Wang and Qingrong Zheng \\ Department of Physics, The University of Hong Kong, Pokfulam Road, Hong Kong
}

Hong Guo

Centre for the Physics of Materials, Department of Physics, McGill University, Montreal, Quebec, Canada H3A 2T8

(Received 20 September 1996; revised manuscript received 22 November 1996)

\begin{abstract}
We have studied the weakly nonlinear quantum transport properties of a two-dimensional quantum wire that can be solved exactly. The nonlinear transport coefficients have been calculated and interesting physical properties revealed. In particular we found that as the incoming electron energy approaches a resonant point given by energy $E=E_{r}$, where the transport is characterized by a complete reflection, the second-order nonlinear conductance changes its sign. We have also investigated the establishment of the gauge-invariance condition. We found that for systems with a finite scattering region, correction terms to the theoretical formalism are needed to preserve the gauge invariance. These corrections were derived analytically for this model. [S0163-1829(97)02916-0]
\end{abstract}

\section{INTRODUCTION}

Nonlinear quantum transport in mesoscopic systems has been a very active research field in recent years. ${ }^{1-4} \mathrm{Ta}$ boryski et al. ${ }^{4}$ have reported observations of nonlinear and asymmetric conductance oscillations of quantum point contacts at a small bias voltage. They found that the non-Ohmic and asymmetric behavior causes a rectified dc signal as the response to an applied ac current. On the theoretical side, Wingreen et al. ${ }^{3}$ have presented a general formulation to deal with the situation of a nonlinear and time-dependent current going through a small interacting region where electron energies can be changed by time-dependent voltages. At the same time, Büttiker and his co-workers ${ }^{5,1,6}$ have advanced a current-conserving theory for the frequencydependent transport. Recently, this current-conserving formalism has been applied to a two-dimensional mesoscopic conductor. $^{7}$ This theory can also be applied to discuss the nonlinear behavior of mesoscopic samples and the theory is gauge invariant. It has been recognized ${ }^{8}$ that in nonlinear coherent quantum transport, it is essential to consider the internal self-consistent potential in order to satisfy the gaugeinvariant condition. This condition demands that all physical properties predicted by a theory cannot change if there is a global voltage shift. Obviously this is a fundamental requirement.

Recently, Christen and Büttiker ${ }^{8}$ have investigated the rectification coefficient of a quantum point contact and the nonlinear current-voltage characteristic of a resonant level in a double-barrier structure using the theory of gauge-invariant nonlinear conductance. Another important application of this theory is to investigate two-dimensional (2D) mesoscopic and ballistic quantum devices that can now be routinely fabricated in many laboratories. Unfortunately, due to a particular technical difficulty, namely, the evaluation of a quantity called sensitivity (see below), so far little is known for the nonlinear conductance in two dimensions. Clearly, an understanding of the 2D situation is very much needed in order to gain further intuition to the coherent transport and to predict the nonlinear characteristics of the variety of 2D nanostructures. The purpose of this paper is to investigate the gaugeinvariant nonlinear transport in a specific two-dimensional system that can be solved exactly. Hence we were able to obtain various relevant physical quantities. Although a general study for an arbitrary 2D system seems difficult, our perspective is that an exactly solved model is valuable since it clearly and unambiguously reveals the physical properties of the nonlinear transport coefficients.

To be specific, we have considered a very simple 2D model that is a quasi-1D ballistic conductor ${ }^{9}$ with a $\delta$ potential confined inside, as shown in Fig. 1(a). Because quantum scattering in this system leads to mode mixing, which is the basic feature of a two-dimensional system, it provides answers to our $2 \mathrm{D}$ problem. In a previous work ${ }^{10}$ we have used this model to study the electric current conservation of the ac transport formalism at the linear conductance level and calculated the important physical quantities such as the global and local partial density of states. In the following we shall extend our calculation to explicitly calculate the secondorder nonlinear conductance $G_{111}$ and $G_{112}$. Due to the gauge-invariant condition (see below), we should have $G_{111}+G_{112}=0$. It turns out that for systems with a finite scattering volume such as those of any numerical calculations, if the global partial density of states (see below) is computed from the energy derivatives of the scattering matrix, we found that a correction term must be added to satisfy the gauge-invariant condition. We have derived these corrections analytically. For this system there is a resonant state with energy $E_{r}$ characterized by a complete reflection, i.e., the reflection coefficient $R=1$ if the $\delta$ potential is attractive. Our results showed that the second-order nonlinear conductance $G_{111}$ changes sign near the resonant point $E_{r}$. This leads to interesting current-voltage characteristics of this system.

The paper is organized as follows. In the next section we shall briefly review the gauge-invariant theory for nonlinear transport put forth by Büttiker. ${ }^{1}$ In Sec. III we will present the solution of the 2D scattering problem. Some of the tech- 


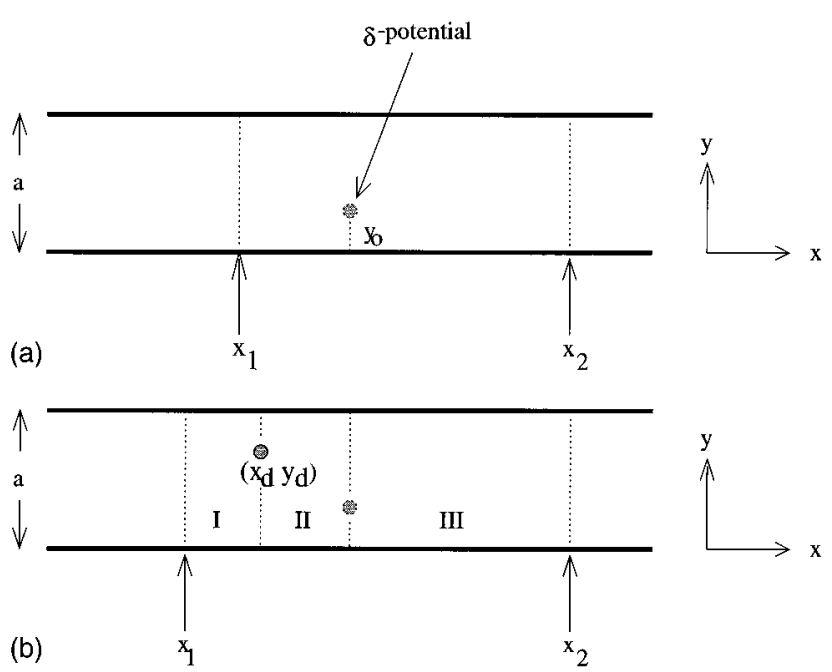

FIG. 1. Schematic plot of the quantum wire system. (a) In the quantum-wire system we have studied a $\delta$ potential $\gamma \delta\left(\vec{r}-\overrightarrow{r_{0}}\right)$ is confined inside a quasi-1D quantum wire, with $\overrightarrow{r_{0}}=\left(0, y_{0}\right)$. The wire width is $a$. The scattering region is between $x_{1}$ and $x_{2}$. In our calculations, the parameters are set to $a=1, y_{0}=0.3, x_{1}=-1$, and $\gamma=-1.0$. (b) To compute the functional derivatives of the scattering matrix with respect to a local potential change, we add another $\delta$-function potential at the position $\left(x_{d}, y_{d}\right)$. In this case the system is divided into three regions by the dotted lines for the boundary matching solution of the Schrödinger equation.

nical details of Sec. III have been put into the Appendix. Our results are presented in Sec. IV. Section V is a brief summary.

\section{GAUGE-INVARIANT FORMALISM}

To be complete, we shall first briefly review the gaugeinvariant formalism of Christen and Büttiker ${ }^{8}$ and present our calculational procedure for the $2 \mathrm{D}$ system. For a multiprobe mesoscopic system, the current through probe $\alpha$ is given by ${ }^{1,8}$

$$
I_{\alpha}=\frac{2 e}{h} \sum_{\beta} \int d E f\left(E-E_{F}-e V_{\beta}\right) A_{\alpha \beta}\left(E,\left\{V_{\gamma}\right\}\right),
$$

where $f(E)$ is the Fermi distribution function and

$$
A_{\alpha \beta}\left(E,\left\{V_{\gamma}\right\}\right)=\operatorname{Tr}\left[\mathbf{1}_{\alpha} \delta_{\alpha \beta}-s_{\alpha \beta}^{\dagger}\left(E,\left\{V_{\gamma}\right\}\right) s_{\alpha \beta}\left(E,\left\{V_{\gamma}\right\}\right)\right]
$$

are the screened (negative) transmission functions that are expressed in terms of the scattering matrix $s_{\alpha \beta}$. For the weakly nonlinear transport, Eq. (1) can be expanded with respect to the voltages $V_{\beta}$,

$$
I_{\alpha}=\sum_{\beta} G_{\alpha \beta} V_{\beta}+\sum_{\beta \gamma} G_{\alpha \beta \gamma} V_{\beta} V_{\gamma}+\cdots,
$$

where

$$
G_{\alpha \beta}=\frac{2 e^{2}}{h} \int d E\left(-\partial_{E} f\right) A_{\alpha \beta}
$$

is the linear conductance and

$$
G_{\alpha \beta \gamma}=\frac{e^{2}}{h} \int d E\left(-\partial_{E} f\right)\left(\partial_{V_{\gamma}} A_{\alpha \beta}+\partial_{V_{\beta}} A_{\alpha \gamma}+e \partial_{E} A_{\alpha \beta} \delta_{\beta \gamma}\right)
$$

is the second-order nonlinear conductance. In Eqs. (4) and (5), the $A_{\alpha \beta}$ are evaluated at $\left\{V_{\gamma}\right\}=0$. The requirements that the current is conserved and independent of a global voltage shift (gauge invariance) yield ${ }^{1,8,11}$

$$
\sum_{\alpha} G_{\alpha \beta}=\sum_{\beta} G_{\alpha \beta}=0
$$

and

$$
\sum_{\alpha} G_{\alpha \beta \gamma}=\sum_{\beta} G_{\alpha \beta \gamma}=\sum_{\gamma} G_{\alpha \beta \gamma}=0 .
$$

From this equation and Eq. (5), the gauge-invariance condition for $A_{\alpha \beta}$ is

$$
e \partial_{E} A_{\alpha \beta}+\sum_{\gamma} \partial_{V_{\gamma}} A_{\alpha \beta}=0 .
$$

Note that the scattering matrix $s_{\alpha \beta}\left(E,\left\{V_{\gamma}\right\}\right)$ is a functional of the electric potential $U\left(\mathbf{r},\left\{V_{\gamma}\right\}\right)$. The derivative $\partial_{V_{\gamma}} A_{\alpha \beta}$ can be expressed in terms of functional derivative of $A_{\alpha \beta}$ with respect the electric potential $U\left(\mathbf{r},\left\{V_{\gamma}\right\}\right)$ and the characteristic potential $u_{\gamma}(\mathbf{r})$,

$$
\partial_{V_{\gamma}} A_{\alpha \beta}=\int d^{3} \mathbf{r} \frac{\delta A_{\alpha \beta}}{\delta U(\mathbf{r})} \frac{\partial U(\mathbf{r})}{\partial V_{\gamma}}=\int d^{3} \mathbf{r} \frac{\delta A_{\alpha \beta}}{\delta U(\mathbf{r})} u_{\gamma}(\mathbf{r}),
$$

where the characteristic potential is defined as ${ }^{1}$

$$
u_{\gamma}(\mathbf{r})=\left(\frac{\partial U(\mathbf{r})}{\partial V_{\gamma}}\right)_{\text {eq }},
$$

where the subscript "eq" denotes the equilibrium value. As a consequence of the gauge invariance, the characteristic potential satisfies the sum rule

$$
\sum_{\gamma} u_{\gamma}(\mathbf{r})=1
$$

To gain further insight into Eq. (6), let us consider a twoprobe system. Equation (3) can be written as

$$
I_{1}=G_{11} V_{1}+G_{12} V_{2}+G_{111} V_{1}^{2}+2 G_{112} V_{1} V_{2}+G_{122} V_{2}^{2} .
$$

Obviously, $G_{12}=-G_{11}$ due to the conservation of electric current. From Eqs. (5)-(7) and (9), we have $G_{111}$ $=-G_{112}=G_{122}$. Therefore, the current depends only on the voltage differences, which is the direct consequence of the gauge-invariant condition Eq. (6). We obtain

$$
I_{1}=G_{11}\left(V_{1}-V_{2}\right)+G_{111}\left(V_{1}-V_{2}\right)^{2} .
$$

For a symmetric system with $U(x, y)=U(-x, y)$, where $x$ is the propagation direction, we must have $-I_{1}$ if $V_{1}$ and $V_{2}$ are interchanged. Hence we conclude that for a symmetric system there are no quadratic terms, i.e., $G_{111}=0$. On the other hand, in general $G_{111} \neq 0$ for geometrically asymmetrical systems. 
To calculate the transmission functions $A_{\alpha \beta}$ and their functional derivatives, we need the characteristic potential $u_{\gamma}$. This in turn needs the solution of the Poisson equation with a nonlocal screening term. ${ }^{1,8}$ To actually carry out this procedure is very complicated. However, if we can use the Thomas-Fermi approximation, which is more appropriate for metallic conductors, the characteristic potential is simplified and is found to be related to the local partial density of states. Within Thomas-Fermi screening, we obtain

$$
u_{\gamma}(\mathbf{r})=\frac{d n(\mathbf{r}, \gamma)}{d E} / \frac{d n(\mathbf{r})}{d E},
$$

where the partial local density of states $d n(\mathbf{r}, \gamma) / d E$ is called the injectivity and is given by ${ }^{11}$

$$
\frac{d n(\mathbf{r}, \gamma)}{d E}=\sum_{n} \frac{\left|\Psi_{\gamma n}\right|^{2}}{h v_{\gamma n}}
$$

where $v_{\gamma n}$ is the channel velocity and $\Psi_{\gamma n}$ is a scattering state. Finally, the quantity $d n(\mathbf{r}) / d E=\Sigma_{\alpha} d n(\mathbf{r}, \alpha) / d E$ is the total local density of states. Substituting Eqs. (2) and (11) into Eq. (7), we obtain

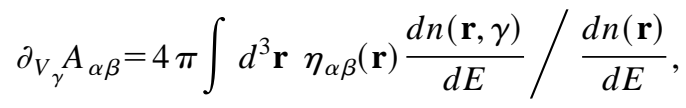

where

$$
\eta_{\alpha \beta}(\mathbf{r})=-\frac{1}{4 \pi} \operatorname{Tr}\left(s_{\alpha \beta}^{\dagger} \frac{\delta s_{\alpha \beta}}{\delta U(\mathbf{r})}+s_{\alpha \beta} \frac{\delta s_{\alpha \beta}^{\dagger}}{\delta U(\mathbf{r})}\right)
$$

is called sensitivity. ${ }^{12}$ We are aware of two ways of calculating the sensitivity. ${ }^{12}$ The first is to evaluate $\delta s_{\alpha \beta} / \delta U$ directly by introducing a $\delta$ function of infinitesimal strength $\delta U$ inside the scattering region. Alternatively, one can calculate it using the retarded Green's function. For a 2D system, in general the Green's function cannot be obtained explicitly; hence we shall use the first method by directly computing the sensitivity. After obtaining the sensitivity, we can then compute $\partial_{V_{\gamma}} A_{\alpha \beta}$ from Eq. (13) and obtain $G_{\alpha \beta \gamma}$ from Eq. (5). Finally, with these explicit results we can directly check the gauge-invariant condition Eq. (6).

\section{MODEL AND ANALYSIS}

As mentioned in the Introduction, Fig. 1(a) shows the system where a $\delta$ potential is confined inside a quasi-1D wire with width $a$. We assume, for simplicity of the calculation, that the boundaries of the ballistic conductor are hard walls, i.e., the potential $V=\infty$ at the walls. Inside the conductor, the potential is zero everywhere except that a $\delta$-function potential $V(x, y)=\gamma \delta(x) \delta\left(y-y_{0}\right)$ is placed at position $\mathbf{r}=\left(0, y_{0}\right)$. The scattering region $x_{1}<x<x_{2}$ is asymmetric, i.e., $x_{1}+x_{2} \neq 0$. From now on we set $\hbar=1$ and $m=1 / 2$ to fix our units.

The transmission and reflection amplitudes have been calculated using a mode-matching method. ${ }^{13,9}$ When the incident electron is in the first subband, in an earlier work we have explicitly obtained these amplitudes. ${ }^{10}$ The evaluation was straightforward but quite tedious; we refer the interested reader to Ref. 10 for details of this algebra. Here we only quote the results: for reflection the amplitude is

$$
b_{n}=\frac{-i \Gamma_{n 1}}{2 k_{n} \alpha}
$$

and for transmission it is

$$
c_{n}=\delta_{n 1}+b_{n} .
$$

Here $\quad \alpha=1+i \Sigma_{n} \Gamma_{n n} / 2 k_{n}, \quad \Gamma_{n m}=\gamma \chi_{n}^{*}\left(y_{0}\right) \chi_{m}\left(y_{0}\right), \quad$ and $\chi_{n}(y)$ is the wave function of the $n$th subband in the $y$ direction. $k_{n}$ is the longitudinal momentum for the $n$th mode given by $k_{n}^{2}=E-(n \pi / a)^{2} ; i=\sqrt{-1}$. Note that for electron traveling in the first subband, $k_{n}$ with $n>1$ is purely imaginary. For our coordinate system the scattering matrix elements $s_{\alpha \beta}$ are given by $s_{11}=b_{1} \exp \left(2 i k_{1}\left|x_{1}\right|\right)$ and $s_{12}$ $=c_{1} \exp \left[i k_{1}\left(x_{2}-x_{1}\right)\right]$.

As mentioned in Sec. II, to calculate the nonlinear conductance of our 2D sample, it is necessary to find the sensitivity $\eta_{\alpha \beta}$. Hence, according to Eq. (14), we must evaluate $\delta s_{\alpha \beta} / \delta U\left(x_{d}, y_{d}\right)$, where the pair $\left(x_{d}, y_{d}\right)$ is an arbitrary location in the scattering volume. For a general 2D sample a direct calculation of this functional derivative is very difficult if not impossible. Fortunately, for our model this can actually be done exactly. As a first step we shall introduce an additional $\delta$ potential of infinitesimal strength $\delta U$ at position $\left(x_{d}, y_{d}\right)$ inside the scattering volume. Thus our system becomes that shown in Fig. 1(b). Then we shall solve the scattering matrix formally as a functional of $\delta U$. Obviously, being able to carry out this step is crucial. Finally, the functional derivative is performed. To proceed we again use the mode-matching method. ${ }^{13,9,10}$ We will assume $x_{d}<0$ in the following calculation. The calculation for $x_{d}>0$ can be done in a similar fashion. The electron wave functions are written as follows. For region I [see Fig. 1(b)]

$$
\Psi_{\mathrm{I}}=\sum_{n} \chi_{n}(y)\left(a_{n} e^{i k_{n} x}+b_{n} e^{-i k_{n} x}\right)
$$

where $a_{n}$ is the incoming wave amplitude and is taken as an input parameter; $b_{n}$ is the reflection amplitude. Similarly, for region II

$$
\Psi_{\mathrm{II}}=\sum_{n} \chi_{n}(y)\left(e_{n} e^{i k_{n} x}+f_{n} e^{-i k_{n} x}\right)
$$

and for region III

$$
\Psi_{\mathrm{III}}=\sum_{n} \chi_{n}(y) c_{n} e^{i k_{n} x}
$$

where $c_{n}$ is the transmission amplitude. We shall match the wave functions and their $x$ derivatives at the positions $x=x_{d}$ and $x=0$. We obtain, at $x=x_{d}$,

$$
a_{n} e^{i k_{n} x_{d}}+b_{n} e^{-i k_{n} x_{d}}=e_{n} e^{i k_{n} x_{d}}+f_{n} e^{-i k_{n} x_{d}}
$$

and

$$
\begin{gathered}
i k_{n}\left(e_{n} e^{i k_{n} x_{d}}-f_{n} e^{-i k_{n} x_{d}}\right)-i k_{n}\left(a_{n} e^{i k_{n} x} d-b_{n} e^{-i k_{n} x_{d}}\right) \\
=\sum_{m} \widetilde{\Gamma}_{n m}\left(e_{m} e^{i k_{m} x_{d}}+f_{m} e^{-i k_{m} x_{d}}\right)
\end{gathered}
$$


where $\widetilde{\Gamma}_{n m}=\delta U \chi_{n}^{*}\left(y_{d}\right) \chi_{m}\left(y_{d}\right)$. At $x=0$, the matching gives

$$
e_{n}+f_{n}=c_{n}
$$

and

$$
i k_{n} c_{n}-i k_{n}\left(e_{n}-f_{n}\right)=\sum_{m} \Gamma_{n m} c_{m} .
$$

To simplify the notation, from now on $\left(x_{d}, y_{d}\right)$ is replaced by $(x, y)$. From the last two equations, we solve for $e_{n}$ and $f_{n}$,

$$
2 i k_{n} e_{n}=-\sum_{m} P_{n m} c_{m}
$$

and

$$
2 i k_{n} f_{n}=\sum_{m} \Gamma_{n m} c_{m}
$$

where $P_{n m}=\Gamma_{n m}-2 i k_{n} \delta_{n m}$. Eliminating $b_{n}$ from Eqs. (17) and (18), we obtain

$$
2 i k_{n} e_{n} e^{i k_{n} x}=2 i k_{n} a_{n} e^{i k_{n} x}+\sum_{m} \widetilde{\Gamma}_{n m}\left(e_{m} e^{i k_{m} x}+f_{m} e^{-i k_{m} x}\right) .
$$

Taking the limit $\delta U \rightarrow 0$, we have

$$
2 i k_{n} \frac{\delta e_{n}}{\delta U} e^{i k_{n} x}=\sum_{m} \bar{\Gamma}_{n m}\left(a_{m} e^{i k_{m} x}+b_{m} e^{-i k_{m} x}\right),
$$

where $\bar{\Gamma}_{n m}=\widetilde{\Gamma}_{n m} / \delta U$. To arrive at the above result we have used the fact that as $\delta U \rightarrow 0$, i.e., when the extra $\delta$ function vanishes, we must have $e_{m}=a_{m}$ and $f_{m}=b_{m}$. From Eq. (19), we have

$$
2 i k_{n} \frac{\delta e_{n}}{\delta U}=-\sum_{m} P_{n m} \frac{\delta c_{n}}{\delta U} .
$$

From Eqs. (21) and (22), we arrive at

$$
-\sum_{m} P_{n m} \frac{\delta c_{m}}{\delta U} e^{i k_{n} x}=\sum_{m} \bar{\Gamma}_{n m}\left(a_{m} e^{i k_{m} x}+b_{m} e^{-i k_{m} x}\right)=\chi_{n}^{*} \Psi,
$$

where $\Psi=\Psi_{\text {I }}$ for $x<0$. From Eq. (23), we have

$$
\frac{\delta c_{l}}{\delta U}=-\sum_{n}\left(P^{-1}\right)_{l n} e^{-i k_{n} x} \chi_{n}^{*} \Psi .
$$

The matrix $P^{-1}$ has been obtained in Ref. 10 and we quote

$$
\left(P^{-1}\right)_{l n}=\frac{i}{2 k_{l}}\left(\delta_{l n}-\frac{i \Gamma_{l n}}{2 k_{n} \alpha}\right) .
$$

From this equation and Eq. (16) we see that for $l=1$, i.e., the first subband, $\left(P^{-1}\right)_{1 n}=i c_{n} / 2 k_{1}$ provided that $\chi_{n}$ is real, which is true in our case. This yields

$$
\frac{\delta c_{1}}{\delta U}=\frac{1}{2 i k_{1}} \sum_{n} c_{n} \chi_{n} e^{-i k_{n} x} \Psi .
$$

Similarly, from Eqs. (17), (19), (20), (22), and (23), we obtain

$$
\begin{aligned}
\frac{\delta b_{n}}{\delta U} & =\frac{\delta e_{n}}{\delta U} e^{2 i k_{n} x}+\frac{\delta f_{n}}{\delta U} \\
& =-\frac{e^{2 i k_{n} x}}{2 i k_{n}} \sum_{m} P_{n m} \frac{\delta c_{m}}{\delta U}+\frac{1}{2 i k_{n}} \sum_{m} \Gamma_{n m} \frac{\delta c_{m}}{\delta U} \\
& =\frac{\sin \left(k_{n} x\right)}{k_{n}} \chi_{n} \Psi+\frac{\delta c_{n}}{\delta U} .
\end{aligned}
$$

When $n=1, \delta b_{1} / \delta U$ becomes

$\frac{\delta b_{1}}{\delta U}=\frac{1}{2 i k_{1}}\left(\chi_{1} e^{i k_{1} x}+\sum_{n} b_{n} \chi_{n} e^{-i k_{n} x}\right) \Psi=\frac{1}{2 i k_{1}} \Psi^{2}$.

Because the scattering matrix elements $s_{11} \sim b_{1}$ and $s_{12} \sim c_{1}$ as mentioned above, with the functional derivatives (26) and (24) we can evaluate $\delta s_{\alpha \beta} / \delta U$ trivially, thus obtaining the sensitivity $\eta_{\alpha \beta}$ of Eq. (14). Then using the prescription discussed at the end of Sec. II, we can obtain all the weakly nonlinear conductances and other quantities of interest. Our results will be presented in Sec. IV.

To end this section of the theoretical analysis, we mention that to check the result of functional derivatives, in the Appendix we shall explicitly calculate a quantity called emissivity $^{1}$ using these functional derivatives. In the absence of a magnetic field, it is known ${ }^{11}$ that emissivity is equal to the injectivity defined in Eq. (12), which we can compute using the wave functions. Indeed, we confirm in the Appendix that these two are equal, thus providing a necessary check to the calculations presented here.

\section{RESULTS}

To obtain numerical results from our analytical formula, for the system of Fig. 1(a) we consider incident electron coming from probe 1 and set $a=1, y_{0}=0.3, x_{1}=-1$, and $\gamma=-1$. Although we have restricted the incoming electron energy to the first subband, quantum scattering at the $\delta$-function potential leads to mode mixing. Thus, in our numerical calculations we have included 50 modes in the scattering volume. We have checked that this is enough to obtain good numerical convergence.

As a first result we plot the sensitivity $\eta_{11}(\mathbf{r}, E)$ as a function of the electron incident energy $E$ at several positions r. This is shown in Fig. 2. As discussed in Sec. II, $\eta_{\alpha \beta}$ appears naturally in the theoretical formalism and it essentially describes the local electric current response of the scattering problem when there is a small local potential change. It is related to the real part of the diagonal elements of the Green's function. ${ }^{12}$ Figure 2 not only shows the interesting behavior of this quantity, but also gives vivid intuition about the local current response. As shown in our earlier work ${ }^{10}$ and mentioned above, in the presence of an attractive $\delta$-function scatterer, the quantum wire studied here has a resonant state at energy $E=E_{r}=36.65$, where we have a complete reflection (reflection coefficient $R=1$ or $s_{11}=0$ ). From Eq. (14) we have $\eta_{11}(\mathbf{r})=0$ at the resonant energy $E=E_{r}$, which can be seen from Fig. 2. Near this resonance energy the system response is very sensitive to potential perturbations and this is signaled by the large peak of $\eta_{11}$. On the other hand, this peak is larger when we are closer to the 


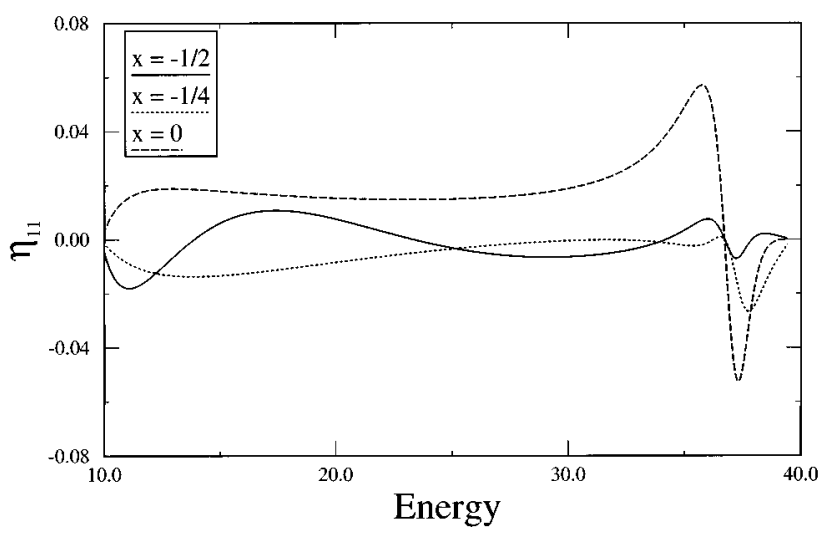

FIG. 2. Sensitivity $\eta_{11}(\mathbf{r}, E)$ as a function of energy $E$ at three different positions $x=-1 / 2,-1 / 4,0$ with the same $y=0.3$. For different $y$ the curve $\eta_{11}$ as a function of $E$ will be multiplied by a constant. Other system parameters are the same as those of Fig. 1. Here the unit of energy is $\hbar^{2} / 2 m a^{2}$.

$\delta$-function scatterer located at $x=0$ : this indicates that the local perturbation has larger effects when it is closer to the scattering center. Although Fig. 2 shows $\eta_{11}$ at positions to the left of the scatterer, we have checked that its behavior is exactly the same for positions $x>0$, i.e., $\eta_{11}(x)$ is an even function of $x$.

Adding up all the local responses according to Eq. (13), we can explicitly examine the gauge-invariant condition (6). Using Eqs. (2) and (7) and the fact that $u_{1}+u_{2}=1$, Eq. (6) reduces to

$$
2 \operatorname{Re}\left(s_{\alpha \beta}^{\dagger} \frac{d s_{\alpha \beta}}{d E}\right)+2 \int d^{3} \mathbf{r} \operatorname{Re}\left(s_{\alpha \beta}^{\dagger} \frac{\delta s_{\alpha \beta}}{\delta U(\mathbf{r})}\right)=0
$$

It is straightforward to evaluate the left-hand side of this equation. Using the functional derivatives obtained in Sec. III, as well as the energy derivatives of Eqs. (15) and (16), we found that the left-hand side of the above equation is nonzero. For instance, for a symmetric system, it is given by

$\mathcal{C}=\frac{\left|s_{12}\right|^{2}}{k_{1}^{2}} \operatorname{Re}\left(s_{11}\right)+\operatorname{Re}\left(\sum_{n=2} \frac{b_{1}\left|b_{n}\right|^{2}}{k_{1} k_{n}} e^{i k_{n}\left(x_{2}-x_{1}\right)}\right)$,

where $\mathcal{C}$ denotes the correction. Thus, in order to have precise gauge invariance, this correction must be included. From this result, we notice that the first correction term is only significant near the first subband threshold where $k_{1} \approx 0$ and is negligible for larger incoming electron energies. For the second correction term, let us examine its behavior near the $n$th subband with $n>1$. From Eq. (15) we see that as the incoming electron momentum $k \rightarrow k_{n}$, $b_{1} \rightarrow k_{n}$, and $b_{n}$ is finite. Therefore, the second correction term remains finite when the electron energy approaches the $n$th subband $(n>1)$. This is different from the ac transport where the correction diverges ${ }^{10}$ near the $n$th subband with $n>1$. We emphasize that the correction term is due to the fact that we are considering a finite scattering volume. As the scattering volume or the incident energy becomes larger, the effect of these correction terms diminishes. This can be seen

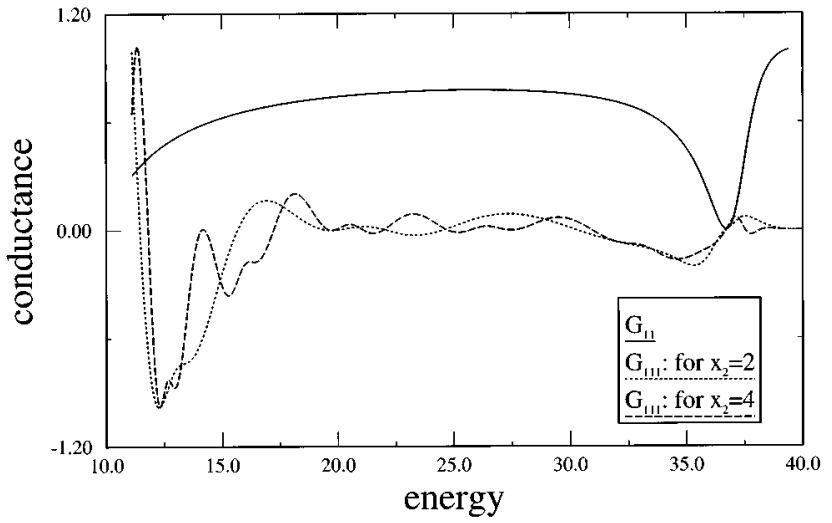

FIG. 3. Conductances $G_{11}$ and $G_{111}$ as functions of energy $E$. Solid line, $G_{11}$; dashed line, $G_{111}$ with $x_{2}=2$; dotted line, $G_{111}$ with $x_{2}=4$. Other system parameters are the same as those of Fig. 1. Here the unit of energy is $\hbar^{2} / 2 m a^{2}$.

clearly due to the factor $k_{1}$ in the denominator and the exponentially decaying factor $\exp \left[i k_{n}\left(x_{2}-x_{1}\right)\right]$ as $k_{n}$ is purely imaginary for all $n>1$.

The origin of this correction term is in the energy derivative of $\partial_{E} A_{\alpha \beta}$ as discussed in Refs. 10 and 12. Although it is nonessential for large scattering volumes, care must be taken when using Eq. (5) in any numerical calculations where the scattering volume is always finite. For instance, if one directly uses Eq. (5) to compute $G_{111}$ for a geometrically symmetric system, a nonzero result will be obtained. Therefore, in principle, one needs to distribute the correction term separately to $G_{111}$ and $G_{112}$ in order to obtain physically correct quantities. Alternatively, for the present problem of evaluating the second-order nonlinear conductances, one can eliminate $\partial_{E} A_{\alpha \beta}$ in Eq. (5) by the gauge-invariance condition (6), which yields

$$
\begin{aligned}
G_{\alpha \beta \gamma}= & \frac{e^{2}}{h} \int d E\left(-\partial_{E} f\right) \\
& \times \int d^{3} \mathbf{r} \frac{\delta A_{\alpha \beta}}{e \delta U(\mathbf{r})}\left[u_{\gamma}(\mathbf{r})+u_{\beta}(\mathbf{r})-\delta_{\gamma \beta}\right],
\end{aligned}
$$

where we have used Eqs. (7) and (11). Since all quantities are computed locally, i.e., in the scattering volume, Eq. (27) gives the correct behavior without the need to distribute the correction terms. $^{14}$

Now we present numerical evaluations of the secondorder nonlinear conductance $G_{\alpha \beta \gamma}$ using Eq. (27) with the help of the analytical expressions for the various quantities involved. In Fig. 3 the second-order nonlinear conductance $G_{111}$ is plotted for two different scattering regions: $x_{2}=2$ (dashed line) and 4 (dotted line). For illustration purposes, we have multiplied $G_{111}$ by a factor of 20 . In comparison, the solid line shows the linear conductance $G_{11}$, which is proportional to the transmission coefficient by the Landauer formula. At the resonance $E=E_{r}=36.65$, we observe the complete reflection ${ }^{15,10}$ indicated by $G_{11}=0$. As expected, the nonlinear coefficient $G_{111}$ also vanishes at $E_{r}$. Furthermore, $G_{111}$ changes its sign as the incoming electron energy varies and has many oscillations for these asymmetrical systems. This has important implications on the current-voltage char- 


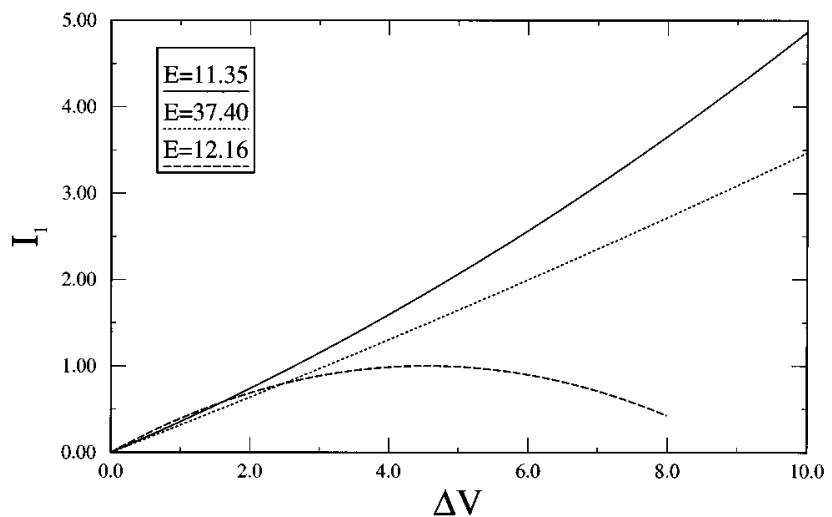

FIG. 4. Current-voltage characteristics as calculated from Eq. (10) at several different electron energies: $E=11.35$ (solid line), 37.40 (dotted line), and 12.16 (dashed line). $x_{2}=2$ is used. $\Delta V=V_{1}-V_{2}$. Other system parameters are the same as those of Fig. 1.

acteristics if we recall the $I-V$ relation Eq. (10). The $I-V$ curves of a system with $x_{2}=2$ is shown in Fig. 4 for several different electron energies. We can clearly see that for a positive $G_{111}$, such as $E=11.35$ (solid line) and 37.40 (dotted line), the current $I_{1}$ increases with the potential difference of the two probes $\Delta V=V_{1}-V_{2}$. However, when $G_{111}$ is negative, such as at $E=12.16$ (dashed line), $I_{1}$ decreases for a range of $\Delta V .^{16}$

\section{SUMMARY}

To summarize, we have solved exactly the weakly nonlinear transport characteristics of a two-dimensional quantum-wire model. To the best of our knowledge, this is the first exact solution for a truly two-dimensional ballistic model. The second-order nonlinear conductances are derived analytically. We found that as the incoming electron energy crosses the resonant point, the nonlinear conductance changes its sign. This leads to interesting current-voltage behavior when the incoming electron energy changes. We have also examined the gauge-invariant condition that is obtained by the global voltage shift. We found that for systems with a finite scattering volume, correction terms are needed to preserve the gauge-invariant condition when we compute the global partial density states using the energy derivative of the scattering matrix. We have derived these corrections analytically for our model. The correction term consists of two parts. The first part dominates when the incident energy $E$ is near the first subband threshold. On the other hand, the second part is given by the amplitudes of the nonpropagating modes and is significant near the resonant point. Finally, our exact calculation reveals the interesting behavior of the sensitivity that describes the local electric current response to a potential perturbation.

\section{ACKNOWLEDGMENTS}

We thank Professor M. Büttiker for pointing out that in our problem $G_{111}$ can be nonzero only if the boundaries are nonsymmetric with respect to the location of the impurity. We gratefully acknowledge support by a RGC grant from the Government of Hong Kong under Grant No. HKU 261/95P, a research grant from the Croucher Foundation, the Natural Sciences and Engineering Research Council of Canada, and le Fonds pour la Formation de Chercheurs et l'Aide à la Recherche de la Province du Québec. We thank the Computer Center of the University of Hong Kong for computational facilities.

\section{APPENDIX}

To check our result of the functional derivatives, i.e., Eqs. (24) and (25), in this appendix we compute the emissivity defined as ${ }^{1}$

$\frac{d n(\alpha, \mathbf{r})}{d E}=-\frac{1}{4 \pi i} \sum_{\beta} \operatorname{Tr}\left[s_{\alpha \beta}^{\dagger} \frac{\delta s_{\alpha \beta}}{\delta U(\mathbf{r})}-\frac{\delta s_{\alpha \beta}^{\dagger}}{\delta U(\mathbf{r})} s_{\alpha \beta}\right]$

It has been shown ${ }^{11}$ that in the absence of a magnetic field the emissivity is equal to the injectivity defined in Eq. (12). We shall explicitly perform the functional derivatives to confirm this fact and hence provide the necessary check to our algebra.

Using Eqs. (24) and (25), we have

$$
\begin{aligned}
s_{11}^{*} \frac{\delta s_{11}}{\delta U}+s_{12}^{*} \frac{\delta s_{12}}{\delta U} \\
=c_{1}^{*} \frac{\delta c_{1}}{\delta U}+b_{1}^{*} \frac{\delta b_{1}}{\delta U} \\
=\frac{1}{2 i k_{1}}\left(c_{1}^{*} \chi_{1} e^{-i k_{1} x}+c_{1}^{*} \sum_{n} b_{n} \chi_{n} e^{-i k_{n} x}\right. \\
\left.\quad+b_{1}^{*} \chi_{1} e^{i k_{1} x}+b_{1}^{*} \sum_{n} b_{n} \chi_{n} e^{-i k_{n} x}\right) \Psi \\
=\frac{1}{2 i k_{1}}\left(b_{1}^{*} \chi_{1} e^{i k_{1} x}+\chi_{1} e^{-i k_{1} x}\right. \\
\left.\quad+\left(1+2 b_{1}^{*}\right) \sum_{n=2} b_{n} \chi_{n} e^{-i k_{n} x}\right) \Psi
\end{aligned}
$$

where the relation $c_{1}=1+b_{1}$ has been used. Before we proceed further, let us derive a useful relation from the unitary condition of the scattering matrix, namely,

$$
1+2 b_{1}^{*}=-\frac{b_{1}^{*}}{b_{1}}=\frac{\alpha}{\alpha^{*}} .
$$

The first equality comes from the unitary condition $c_{1}^{*} b_{1}+c_{1} b_{1}^{*}=0$ or $b_{1}^{*}+\left(1+2 b_{1}^{*}\right) b_{1}=0$; the second equality is from Eq. (15). Since the incoming electron is in the first subband, we have $k_{n}^{*}=-k_{n}$ for $n>1$. Hence, for $n>1$,

$$
\frac{b_{n}}{b_{n}^{*}}=\frac{\alpha^{*}}{\alpha}
$$

Substituting Eqs. (A2) and (A3) into Eq. (A1), we obtain 


$$
s_{11}^{*} \frac{\delta s_{11}}{\delta U}+s_{12}^{*} \frac{\delta s_{12}}{\delta U}=\frac{1}{2 i k_{1}}|\Psi|^{2},
$$

which is equivalent to Eq. (12). Notice that the imaginary part of the left-hand side of Eq. (A4) is proportional to the emissivity. Its real part gives the sensitivity $\eta_{11}+\eta_{12}$. From the unitary condition we have $\eta_{11}+\eta_{12}=0$, which agrees with Eq. (A4).
${ }^{1}$ M. Büttiker, J. Phys. Condens. Matter 5, 9361 (1993).

${ }^{2}$ B. L. Al'tshuler and D. E. Khmelnitskii, Pis'ma Zh. Éksp. Teor. Fiz. 42, 291 (1985) [JETP Lett. 42, 359 (1985)].

${ }^{3}$ N. S. Wingreen, A. P. Jauho, and Y. Meir, Phys. Rev. B 48, 8487 (1993).

${ }^{4}$ R. Taboryski, A. K. Geim, M. Persson, and P. E. Lindelof, Phys. Rev. B 49, 7813 (1994).

${ }^{5}$ M. Büttiker, A. Prêtre, and H. Thomas, Phys. Rev. Lett. 70, 4114 (1993).

${ }^{6}$ M. Büttiker, Phys. Rev. B 46, 12485 (1992).

${ }^{7}$ Jian Wang and Hong Guo, Phys. Rev. B 54, R11 090 (1996).

${ }^{8}$ T. Christen and M. Büttiker, Europhys. Lett. 35, 523 (1996).

${ }^{9}$ P. F. Bagwell, Phys. Rev. B 41, 10354 (1990).

${ }^{10}$ Jian Wang, Qingrong Zheng, and Hong Guo, Phys. Rev. B 55, 9770 (1997).

${ }^{11}$ M. Büttiker and T. Christen, in Quantum Transport in Semicon- ductor Submicron Structures, edited by B. Kramer (Kluwer Academic, Dordrecht, 1996).

${ }^{12}$ V. Gasparian, T. Christen, and M. Büttiker, Phys. Rev. A 54, 4022 (1996).

${ }^{13}$ R. L. Schult, D. G. Ravenhall, and H. W. Wyld, Phys. Rev. B 39, 5476 (1989).

${ }^{14}$ Clearly, since in deriving Eq. (27) we have already applied the gauge-invariance condition of Eq. (6), there is no need for any correction terms for the $G_{\alpha \beta \gamma}$ if computed using Eq. (27).

${ }^{15}$ Jian Wang, Yongjiang Wang, and Hong Guo, Appl. Phys. Lett. 65, 1793 (1994).

${ }^{16}$ For a positive $V_{1}-V_{2}$ and negative $G_{111}$, Eq. (10) may seem to produce a negative $I_{1}$ if $V_{1}-V_{2}$ is large enough. However, we should keep in mind that there are other higher-order terms not included in Eq. (10) that become important at high-voltage differences. Including all terms will produce a positive current. 